\title{
The Humanistic and Economic Burden of Migraine in Europe: A Cross-Sectional Survey in Five Countries
}

\author{
Michael J. Doane · Shaloo Gupta · Juanzhi Fang · Annik K. Laflamme •
}

Pamela Vo

Received: March 3, 2020 / Published online: June 15, 2020

(C) The Author(s) 2020

\begin{abstract}
Introduction: Prior studies have estimated the burden of migraine in patients suffering from $\geq 4$ monthly headache days (MHDs), but the burden experienced by migraineurs suffering from one to three (1-3) MHDs is unknown. The aim of this study was to examine the incremental burden of migraine in terms of healthrelated quality of life (HRQoL), impairments to work and daily activities, and healthcare resource utilization (HRU) in five European countries (France, Germany, Italy, Spain, and
\end{abstract}

Digital Features To view digital features for this article go to https://doi.org/10.6084/m9.figshare.12311432.

Electronic supplementary material The online version of this article (https://doi.org/10.1007/s40120020-00196-2) contains supplementary material, which is available to authorized users.

M. J. Doane

Kantar, New York, NY 10010, USA

S. Gupta $(\bowtie)$

Kantar, 3 World Trade Center, 35th Floor, New

York, NY 10007, USA

e-mail: shaloo.gupta@kantar.com

J. Fang

Novartis Pharmaceuticals Corporation, One Health

Plaza, East Hanover, NJ 07936, USA

A. K. Laflamme · P. Vo

Novartis Pharma AG, Fabrikstr. 12, 4002 Basel, Switzerland the UK (EU5]), by comparing migraineurs with $\geq 4$ MHDs and migraineurs with 1-3 MHDs.

Methods: The sample for this retrospective cross-sectional study was collected from the 2017 National Health and Wellness Survey $(N=62,000)$. The Short-Form 12-Item Health Survey Instrument, version-2 physical and mental component summary (PCS and MCS) scores, Short-Form 6-dimensions (SF-6D), EuroQoL 5-dimensions (EQ-5D) and EuroQoL visual analog scale (VAS) scores, impairments to work productivity and daily activities (Work Productivity and Activity Impairment [WPAI] Questionnaire) scores, and HRU were compared between migraineur groups with $\geq 4$ MHDs (4-7, intermediate-frequency episodic migraine; 8-14, high-frequency episodic migraine; $\geq 15$ chronic migraine) and the migraineur subgroup with 1-3 MHDs (low-frequency episodic migraine) using generalized linear modeling after adjusting for covariates.

Results: Data from a total of 62,000 survey respondents were examined, of whom 1323 and 1569 were considered to have 1-3 MHDs and $\geq 4$ MHDs (4-7 MHDs [ $n=783$ ]; 8-14 MHDs $[n=429] ; \geq 15$ MHDs $[n=357])$, respectively. The adjusted HRQoL was significantly lower in the 4-7 MHDs (for MCS and SF-6D scores; $p<0.0001$ ) and 8-14 MHDs subgroups (for MCS, SF-6D, EQ-5D, and EuroQoL VAS scores, $p<0.0001$; for PCS scores, $p=0.0007$ ) than in the 1-3 MHDs subgroup. Migraineurs with 4-7 and 8-14 MHDs reported higher activity 
impairment and more frequent visits to healthcare providers (all $p<0.0001$ ) and neurologists $(p=0.0006$ and $p<0.0001$, respectively) compared to the 1-3 MHDs subgroup. Migraineurs with $\geq 15$ MHDs had significantly lower HRQoL and increased WPAI scores and HRU than the 1-3 MHDs subgroup.

Conclusions: This study provides evidence supporting the incremental burden of migraine, characterized by poorer HRQoL and increased WPAI scores and greater HRU, among migraineurs experiencing $\geq 4$ MHDs compared with migraineurs experiencing 1-3 MHDs in the EU5.

Keywords: Activity impairment; Healthcare resource; Health-related quality of life; Migraine; Work impairment

\section{Key Summary Points}

\section{Why carry out this study?}

Increase in the number of monthly headache days (MHDs) can immensely impact functional abilities in patients with migraine.

The majority of migraine patients experience low-frequency episodic attacks (1-3 MHDs), but the burden of migraine experienced by this group is unknown.

We aimed to examine the humanistic and economic burden among migraineurs experiencing $\geq 4 \mathrm{MHDS}$ (categorized in subgroups of $4-7,8-14$, and $\geq 15$ MHDs, respectively) compared with those experiencing 1-3 MHDs, in terms of health-related quality of life (HRQoL), work productivity and activity impairment (WPAI), and healthcare resource utilization (HRU) in five European countries (EU5).

\section{What was learned from the study?}

Among respondents, those experiencing 1-3 MHDs were more prevalent than those experiencing $\geq 4$ MHDs (4-7, 8-14, and $\geq 15$ MHDs).
However, the incremental burden due to migraine in terms of reduced HRQoL, greater work productivity loss and activity impairment, and increased HRU was higher among those in the $\geq 4$ MHDs subgroups than among those in the $\leq 3$ MHDs subgroup.

Respondents experiencing $\geq 4$ MHDs, chronic migraineurs ( $\geq 15$ MHDs) in particular, should be treated more effectively to reduce migraine frequency and lessen the burden of migraine in the EU5.

\section{INTRODUCTION}

Migraine is a primary headache disorder characterized by episodic attacks that has the ability for progression to chronic migraine [1-3]. Migraine affects more than one in ten people worldwide, with the prevalence in Europe (11.4\%) closely mirroring the global prevalence (11.6\%) [4]. Generally beginning around puberty, migraine is most prevalent among adults aged 35-45 years and twice more common in women than men [5]. The Global Burden of Disease 2015 Study reported migraine as the leading cause of years lived with disability worldwide in adults aged 15-49 years, indicating that the burden of migraine is particularly high during the prime years of productivity [6]. Therefore, migraine and an increase in the number of monthly headache days (MHDs) can have an immense impact on the functional abilities of the affected population $[7,8]$.

In one epidemiological survey of migraine, $63 \%$ of respondents had one to four attacks per month [9]. Previous research has examined the impact of episodic ( $<15$ MHDs) and chronic ( $\geq 15$ MHDs) migraine on the health-related quality of life (HRQoL), work productivity and activity impairment (WPAI), and healthcare resource utilization (HRU) of migraineurs across five European (EU5) countries (France, Germany, Italy, Spain, and the UK) [10-13]. 
Although the burden of migraine in individuals suffering from $\geq 4$ MHDs is relatively greater than in those suffering from $\leq 3$ MHD, the prevalence of the former group is lower [14]. Differences in the burden of migraine between those with episodic migraine and those with chronic migraine have been reported earlier $[8,14]$, but only limited data are available on the burden of migraine as a function of the frequency of headache days, particularly among those with one to three MHDs (1-3 MHDs). Moreover, the burden of migraine experienced by those with intermediate-frequency episodic (4-7 MHDs) migraine, high-frequency episodic (8-14 MHDs) migraine, and chronic ( $\geq 15$ MHDs) migraine compared with those experiencing low-frequency (1-3 MHDs) migraine in the EU5 has not been reported earlier.

We hypothesized that increased frequency of migraine or increased MHDs would inflict additional burden on the health, work life, and daily activities of migraineurs. The objective of this study was to characterize this additional burden of migraine with increased MHDs among migraineurs experiencing $\geq 4$ MHDS (4-7, 8-14, and $\geq 15$ MHDs) compared with those experiencing 1-3 MHDs, in terms of HRQOL, WPAI, and HRU in the EU5.

\section{METHODS}

\section{Sample}

This retrospective cross-sectional study used data from the 2017 National Health and Wellness Survey (NHWS; $N=62,000$ ) in the EU5. Respondents were aged $\geq 18$ years, consented to participate in the survey, and could read and write in the primary language of their country of residence (French, German, Italian, Spanish, and English for France, Germany, Italy, Spain, and the UK, respectively) at the time of the survey.

The respondents of this survey were members of MySurvey.com or its partners, which are opt-in survey panels. They were recruited through opt-in e-mail, co-registration with MySurvey.com partners, e-newsletter campaigns, banner placements, and both internal and external affiliate networks. Telephone recruitment using quota sampling, based on age and gender, was used to supplement online recruitment in countries with insufficient internet penetration among the elderly (Spain and Italy) to provide an adequate sample of the elderly population. Those without access to the internet were invited to complete the survey using a computer in a private center. The protocol and questionnaire for the NHWS were reviewed by the Pearl Institutional Review Board and determined to be exempt from review for this study.

The 2017 NHWS was divided into two components: a base survey component that assessed demographics, diseases experienced and diagnosed, and health outcomes (completed by all respondents) and various disease (e.g., migraine) and non-disease (e.g., vaccination) modules completed by eligible respondents. Those who indicated experiencing migraine were assigned to the corresponding condition module $(n=13,063)$, out of which 6181 reported a physician diagnosis of migraine. The latter group completed additional survey questions on the frequency of migraines or headache days and were classified as (1) low-frequency episodic migraine (1-3 MHDs per month), (2) intermediate-frequency episodic migraine (4-7 MHDs per month), (3) high-frequency episodic migraine (8-14 MHDs per month), and (4) chronic migraine ( $\geq 15$ MHDs per month) [11]. The recall period for headache days was the 30 days immediately preceding the day of survey participation. Respondents who either reported not experiencing any migraines in the past month or those who did not know the number of headache days experienced in the prior month were excluded from the analyses $(n=3289)$. In order to study the consequence of migraine frequency on the burden of migraine, physician-diagnosed migraine respondents who had $\geq 4$ MHDs ( $n=1569$ ) were compared based on their frequency of MHDs to those who reported low-frequency episodic migraine $(\leq 3$ MHDs; $n=1323$ ) (Fig. 1). 


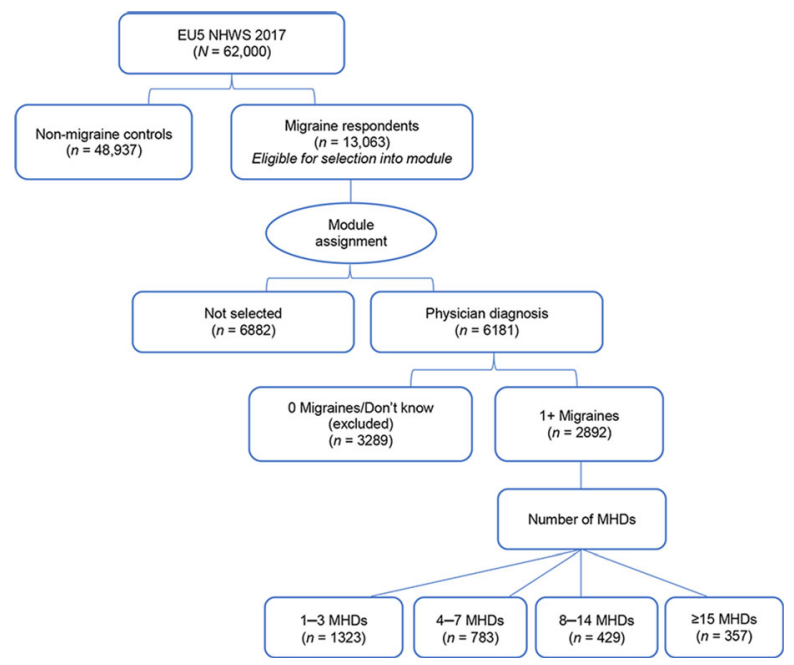

Fig. 1 Flow chart of selection of study population. EUS France, Germany, Italy, Spain, United Kingdom, $M H D s$ monthly headache days, NHWS National Health and Wellness Survey

\section{Measures}

\section{Sociodemographic Characteristics}

Measures included gender, country of residence, age, employment status, annual household income, marital status, and level of education.

\section{General Health Characteristics}

Measures of general health characteristics included body mass index (BMI), cigarette smoking, frequency of alcohol use, days of exercise in the past 30 days, and the Charlson Comorbidity Index (CCI) (the greater the total CCI score, the greater the comorbid burden on the patient) [15].

\section{Health-Related Quality of Life}

Short Form Health Survey 12-v2 The 2017 NHWS included the standard 4-week recall period of the revised Medical Outcomes Study 12-Item Short-Form Health Survey Instrument (SF-12v2), a multipurpose, generic health status instrument comprising 12 questions. Two summary scores were calculated: the physical component summary score (PCS) and the mental component summary (MCS) score. The raw scale scores are transformed to a range from 0 to 100 , with 0 being the worst possible score and 100 being the best possible score $[16,17]$. Higher scores indicate better HRQoL.

The SF-12v2 can also be used to generate health state utilities through the application of the Six-Dimensional Health State Short Form (SF-6D). The SF-6D, which is derived from items on the SF-12v2, is a preference-based, singleindex measure for health using the UK general population values [18]. The SF-6D index has interval scoring properties and yields summary scores six dimensions of health on a theoretical scale of $0-1$, where 1 corresponds to full health and 0 corresponds (in terms of preference) to death [19]. Higher scores indicate better HRQoL. Several studies have used Short Form health surveys to estimate the health status of migraineurs, thus validating the instrument [8].

Measuring HRQoL Using the EuroQol 5 Dimensions Index Score The EuroQol 5 dimensions (EQ-5D) index score is a preferencebased measure of health, ranging on a theoretical scale from 0 to 1 (with1 being equal to full health and 0 being equal [in terms of preference] to death). The most recent version with 5-point rating scales for each dimension was included in the questionnaire for this study (EQ-5D-5L) [20]. The index score for the UK was calculated according to the standard value set for the UK, and the index scores for France, Germany, Italy, and Spain were calculated using the crosswalk from the EQ-5D-3L as no value set for the $5 \mathrm{~L}$ version has been published yet.

The EQ-5D-5L also comprises the EuroQol visual analog scale (EQ-VAS) that allows respondents to self-rate and record their health on a VAS from 0 (representing the worst possible health state) to 100 (representing the best possible health state).

Work Productivity Loss and Activity Impairment Work productivity loss and activity impairment was assessed using the General Health version of the WPAI questionnaire [21], a 6-item validated instrument that consists of four metrics: absenteeism (i.e., defined as employee's absence from work due to their health); presenteeism, (i.e., defined as although the employee may be physically at work, he/she may not be fully functional due to health 
problems); overall work productivity loss; and activity impairment. The outcomes are expressed as percentages of impairment, with higher numbers indicating greater impairment and less productivity [22]. Only respondents who reported being full-time, part-time, or self-employed provided data for absenteeism, presenteeism, and overall work impairment. All respondents completed the activity impairment items.

\section{Healthcare Resource Utilization}

Healthcare resource utilization was defined as the number of visits to different healthcare providers (HCPs) during the last 6 months [8]. All traditional HCP visits, such as primary care provider visits (physicians and nurses), allergist visits, dermatologist visits, and emergency room (ER)/urgent care visits, and hospitalizations were summarized and analyzed for each type of provider as the presence versus absence of a visit in the last 6 months as well as the number of visits during that time.

\section{Statistical Methods}

Data management and analyses were performed using SPSS version 23.0 (IBM Corp., Armonk, NY, USA) and SAS version 9.4 (SAS Institute, Cary, NC, USA). The reporting of descriptive statistics includes frequencies and percentages for categorical variables and means and standard deviations/standard errors for continuous variables. The Chi-square test was used for categorical variables, whereas the Mann-Whitney and $t$ tests were used for continuous variables for the bivariate analysis. Results from the bivariate analysis were used to inform the multivariable regression models. For multivariable analyses, generalized linear models (GLMs) were used to examine the adjusted differences across the migraine subgroups after controlling for the following covariates: sex, country, employment status, household income, education, BMI, smoking status, alcohol use, exercise, age in years, and CCI. GLMs specifying a normal distribution and identity function were used with normally distributed outcomes (such as HRQoL) [23], whereas GLMs specifying a negative binomial distribution and log-link function were used with highly positively skewed variables, such as WPAI [24] and HRU [25], respectively.

\section{RESULTS}

There were a total of 62,000 survey respondents, of whom 1323 and 1569 were considered to have 1-3 MHDs and $\geq 4$ MHDs (4-7 MHDs $[n=783] ; 8-14$ MHDs $[n=429] ; \geq 15$ MHDs $[n=357]$ ), respectively (Fig. 1 ).

Migraineurs were categorized into subgroups based on MHDs experienced during the prior month: 1-3 MHDs (45.8\%), 4-7 MHDs (27.1\%), 8-14 MHDs (14.8\%), and $\geq 15$ MHDs (12.3\%). The majority of respondents were female across all groups $(>70 \%)$. Significant differences in age, household income, education, alcohol consumption, exercise, and CCI were observed between respondents with 1-3 MHDs and $\geq 15$ MHDs. Other demographic and clinical characteristics are shown in Table 1.

\section{Bivariate Results}

\section{Health-Related Quality of Life}

The HRQoL was lower in all of the $\geq 4 \mathrm{MDHs}$ subgroups, including the 4-7 MHDs (for MCS and SF-6D scores, $p<0.001$; for EQ-5D and EQVAS scores, $p<0.01$ ), the 8-14 MHDs, and the $\geq 15$ MHDs subgroups (for all parameters, $p<0.001$ for both subgroups; see Electronic Supplementary Material [ESM] Table S1), compared with the 1-3 MHDs subgroup.

\section{Work Productivity and Activity Impairment}

The employed respondents in the 4-7 MHDs subgroup reported higher activity impairment compared with those in the 1-3 MHDs subgroup $(p<0.001)$, while those in the 8-14 MHDs subgroup reported higher absenteeism, presenteeism, total work productivity impairment (for all subgroups, $p<0.01$ ), and activity impairment $(p<0.001)$ compared to the $1-3$ MHDs subgroup. The $\geq 15$ MHDs subgroup reported significantly greater impairment on all 


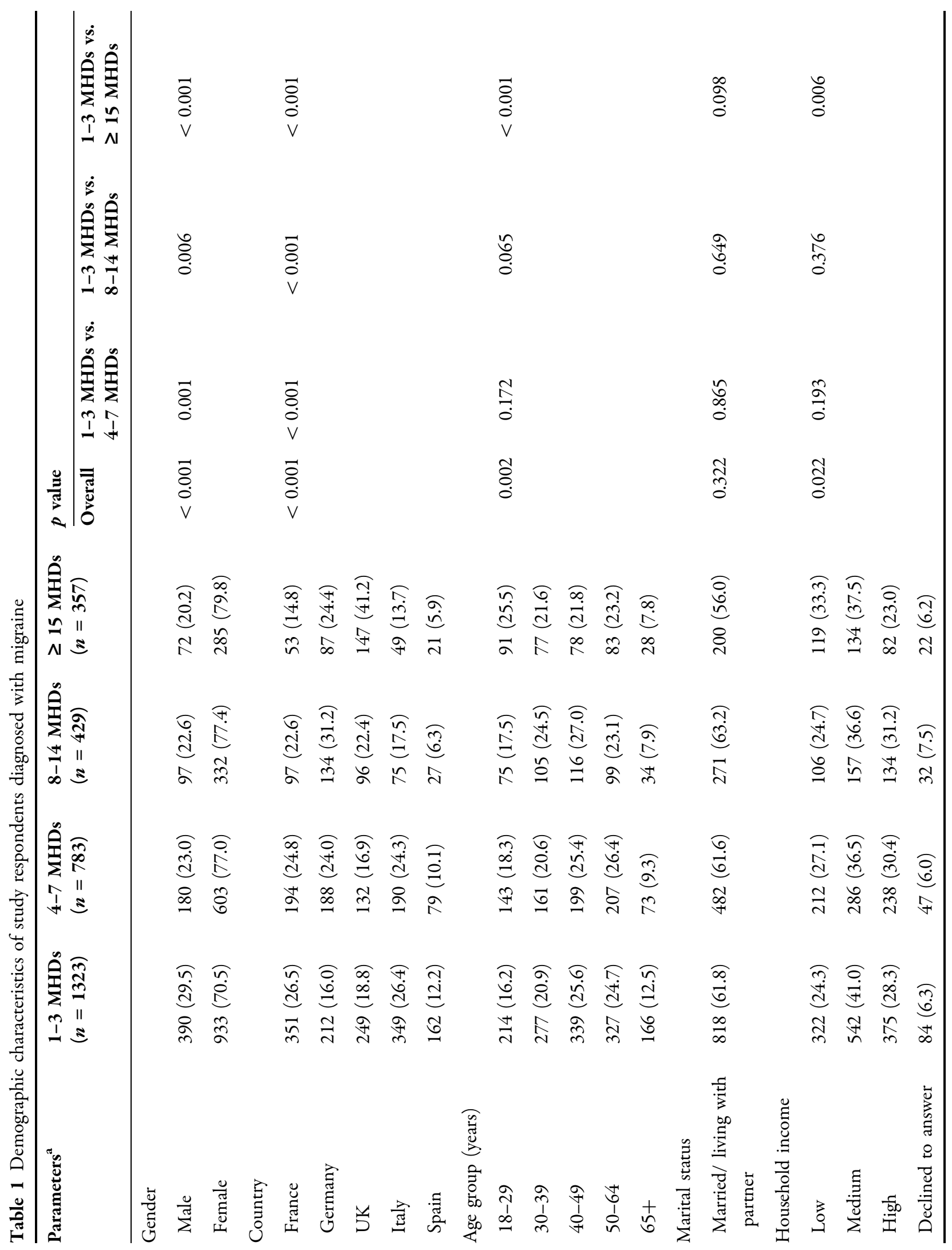




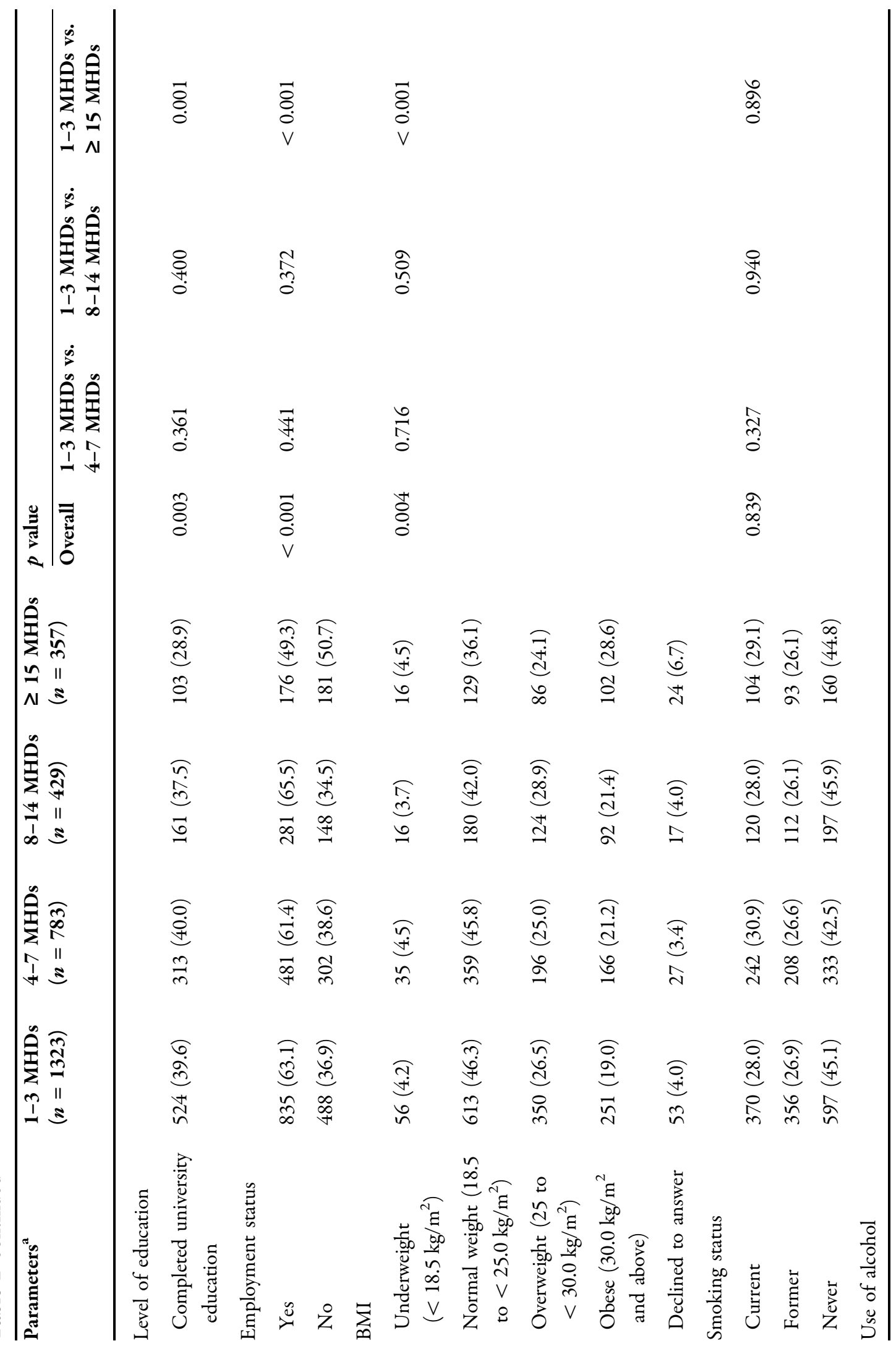




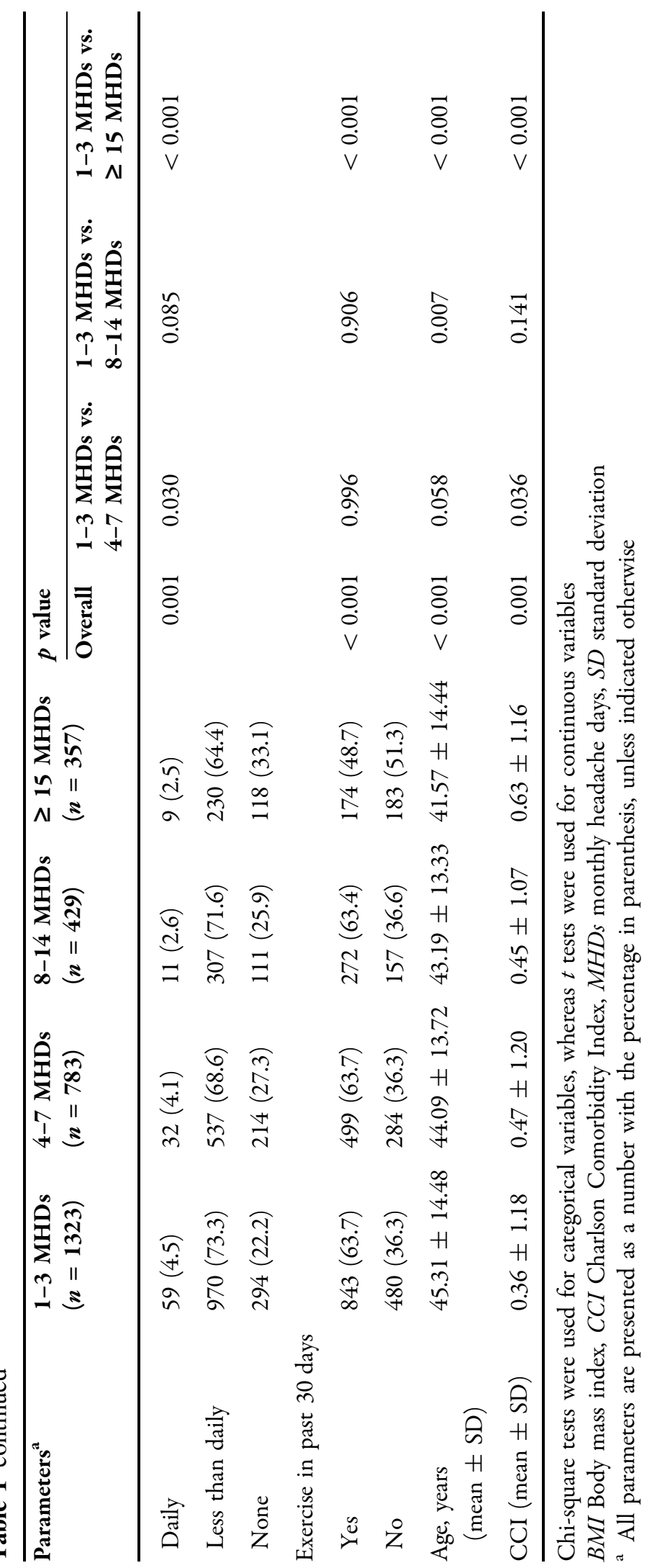


four WPAI metrics $(p<0.001)$ compared to the 1-3 MHDs subgroup (EMS Table S1).

\section{Healthcare Resource Utilization}

A significantly higher number of visits to HCPs and neurologists (for both, $p<0.001$ ) were observed in all of the $\geq 4$ MHDs subgroups compared with the 1-3 MHDs subgroup. Compared with the 1-3 MHDs subgroup, the number of respondents reporting at least one ER visits was significantly higher for the 4-7 $(p<0.01)$ and $\geq 15$ MHDs $(p<0.001)$ subgroups, and hospitalization was significantly greater for the 8-14 and $\geq 15$ MHDs $(p<0.01)$ subgroups (Table ESM S1).

\section{Adjusted Results}

\section{Health-Related Quality of Life}

Migraineurs who reported 4-7 MHDs had significantly lower HRQoL than did those in the 1-3 MHDs subgroup (for MCS and SF-6D scores, $p<0.0001)$. Similarly, migraineurs in the the 8-14 and $\geq 15$ MHDs subgroups reported significantly lower HRQoL compared with the 1-3 MHDs subgroup (for MCS, PCS, SF-6D, EQ-5D, and EQ-VAS scores, $p<0.0001$; except for PCS score of 8-14 MHDs, where $p=0.0007$ ) (Fig. 2).

\section{Work Productivity Loss and Activity Impairment}

Employed migraineurs in the 4-7 and 8-14 MHDs subgroups showed significantly greater activity impairment compared with those in the 1-3 MHDs subgroup ( $p<0.0001$, for both). In addition, the $\geq 15$ MHDs subgroup reported significantly greater absenteeism $(p=0.004)$, presenteeism $(p<0.0001)$ and WPAI $(p<0.0001)$ compared with the 1-3 MHDs subgroup (Fig. 3).

\section{Healthcare Resource Utilization}

Migraineurs with 4-7, 8-14, and $\geq 15$ MHDs had a significantly higher number of total HCP visits than the 1-3 MHDs subgroup (all, $p<0.0001$; Table 2). The total number of visits to the neurologist were significantly higher in the 4-7 $(p=0.001), 8-14$, and $\geq 15$ MHDs $(p<0.0001$, for both) subgroups than in the $1-3$
MHDs subgroup. Additionally, the numbers of total visits to the ER $(p<0.001)$ and psychologists ( $p=0.004$ ) were significantly higher in the $\geq 15$ MHDs subgroup compared to the $1-3$ MHDs subgroup.

\section{DISCUSSION}

This study describes multiple dimensions of patient-reported outcomes, including HRQoL, WPAI, and HRU, in migraineurs experiencing $4-7,8-14$, and $\geq 15$ MHDs compared with those experiencing 1-3 MHDs in EU5. When study respondents were categorized based on number of MHDs, the percentage of patients experiencing 1-3 MHDs (45.7\%) was the highest, followed by those experiencing 4-7 (27.1\%), 8-14 (14.8\%), and $\geq 15$ MHDs (12.3\%). However, the burden associated with migraine in terms of poorer HRQoL and increased WPAI and HRU was higher in those experiencing chronic migraine ( $\geq 15$ MHDs) followed by those experiencing high-frequency (8-14 MHDs) and intermediate-frequency (4-7 MHDs) migraines, compared with those experiencing low-frequency migraine (1-3 MHDs), both before and after adjusting for the confounders.

The poorer HRQoL observed in our study with increasing MHDs is consistent with the findings from a previous study that showed poorer HRQoL in terms of lower mental, physical, and overall health status among migraineurs experiencing $\geq 4$ MHDs, especially in those experiencing $\geq 15$ MHDs, compared with non-migraine controls [8]. In the current study, there were greater levels of absenteeism, presenteeism, and total work productivity impairment in the $\geq 4$ MHDs subgroups than in the 1-3 MHDs subgroup, which is consistent with results from previous studies $[8,26]$.

In addition to impaired HRQoL and greater work productivity loss and activity impairment, migraine also imposes a greater burden on society and on the healthcare system due to an increased consumption of healthcare resources. In this study, HRU was significantly greater in the $\geq 4$ MHDs subgroups than in the 1-3 MHDs subgroup. These findings are consistent with earlier research in Europe and the USA showing 

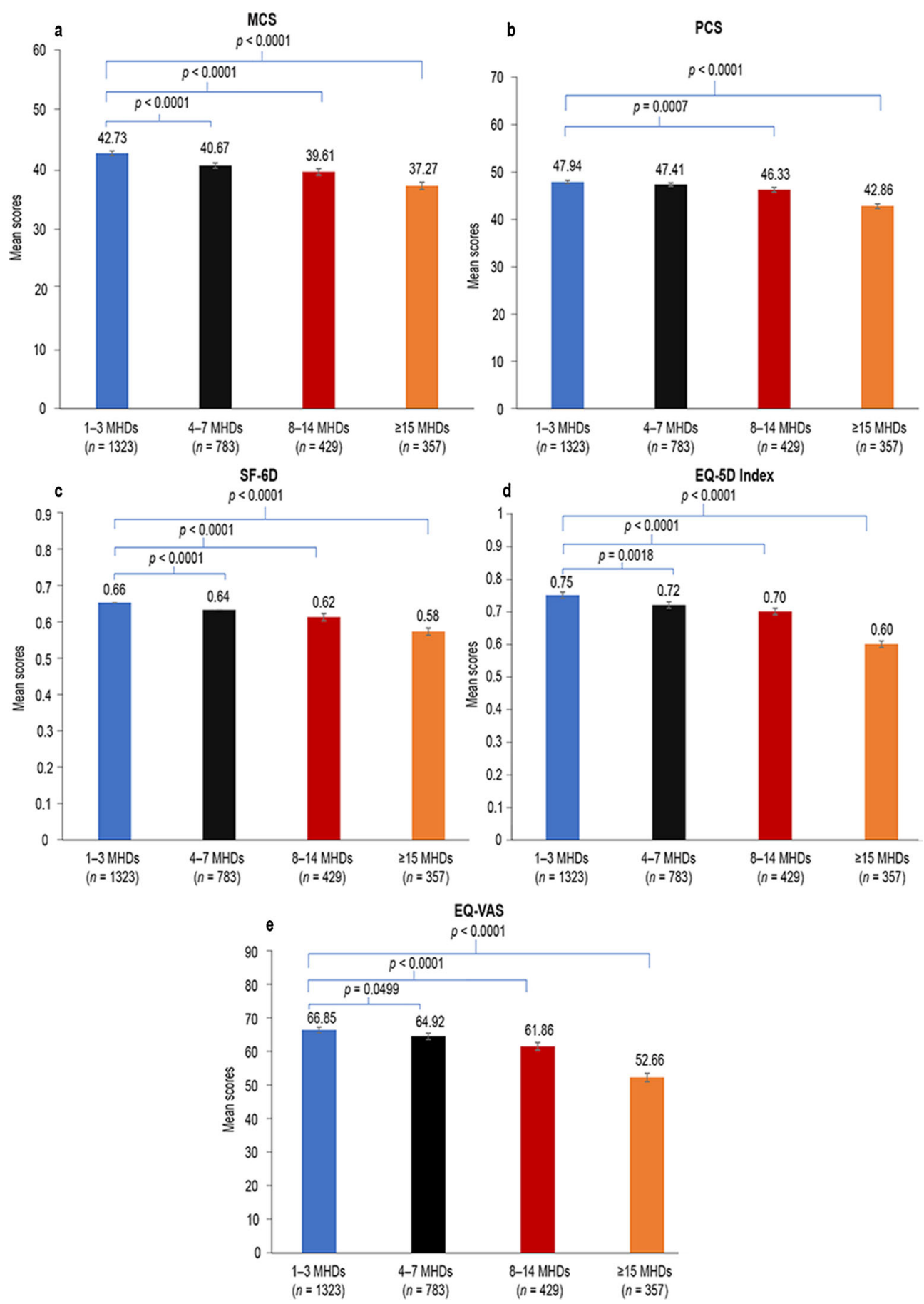

Fig. 2 Health-related quality of life instrument scores. a, b Mental component summary $(M C S ; \mathbf{a})$ and physical component $(P C S ; \mathbf{b})$ scores of the Short Form Health Survey 12-v2, c Six-Dimensional Health State Short Form $(S F-6 D)$ health utility scores, d EuroQol 5 dimensions
$(E Q-5 D)$ index score, and e EuroQoL visual analog scale (EQ-VAS) scores for the $\geq 4$ MHDs subgroups vs. the 1-3 MHDs subgroup. Significance ( $p$ ) levels between subgroups is indicated 

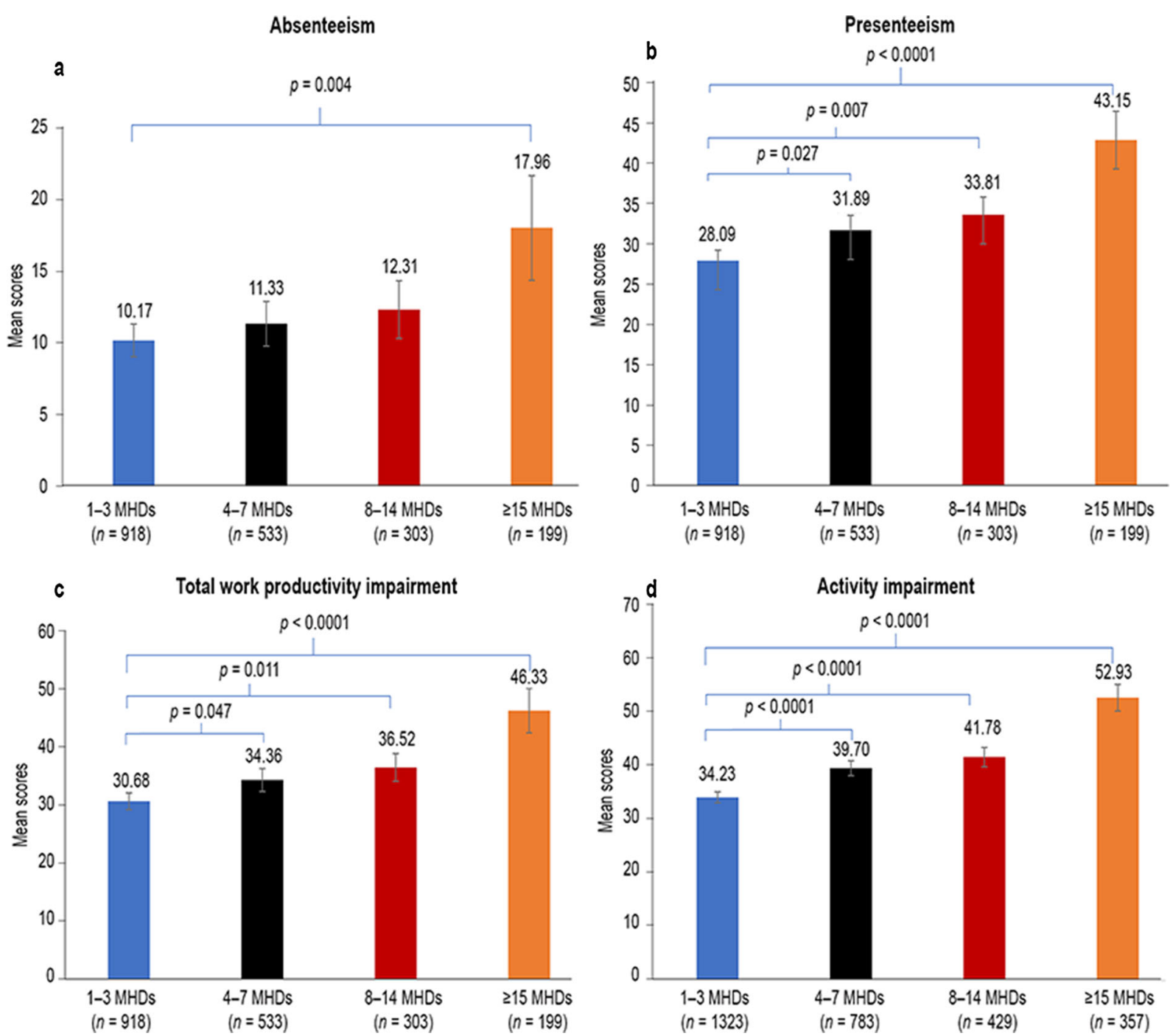

Fig. 3 Work productivity loss and activity impairment assessment. a Absenteeism and b presenteeism. c total work productivity impairment, and $\mathbf{d}$ activity impairment for $\geq 4$ MHDs subgroups vs. the 1-3 MHDs subgroup

that migraineurs, particularly chronic migraineurs, visited physicians, general practitioners, psychiatrists, and neurologists more frequently than did non-migraine controls and episodic migraineurs $[8,10,27]$. Therefore, increased migraine frequency is associated with increased HRU.

A recent study by $\mathrm{Vo}$ et al. [8] among migraineurs in the EU5 reported that migraineurs with $\geq 4$ MHDs, particularly chronic migraineurs (>15 MHDs), had lower HRQoL, decreased work productivity, and increased activity impairment, and greater HRU compared to non-migraine controls. The current study revealed a high impact of migraine in all of the $\geq 4$ MHDs subgroups compared with the 1-3 MHDs subgroup. Although the previous study showed an increased burden in migraineurs with $\geq 4$ MHDs (particularly among those with 8-14 and $\geq 15$ MHDs) compared with non-migraine controls using a smaller sample size [8], the current study uses a larger sample size and reveals that there is an incremental burden due to migraine in all of the subgroups, including the 4-7 MHDs subgroup, but compared with 1-3 MHDs group. Therefore, this 
Table 2 Multivariable analyses of healthcare resource utilization in the past 6 months among migraine subgroups

\begin{tabular}{|c|c|c|c|c|}
\hline Parameters $($ mean \pm SE) & $\begin{array}{l}\text { 1-3 MHDs } \\
(n=1323)\end{array}$ & $\begin{array}{l}\text { 4-7 MHDs } \\
(n=783)\end{array}$ & $\begin{array}{l}\text { 8-14 MHDs } \\
(n=429)\end{array}$ & $\begin{array}{l}\geq 15 \text { MHDs } \\
(n=357)\end{array}$ \\
\hline Total number of HCP visits & $6.08 \pm 0.25$ & $7.57 \pm 0.36^{\dagger}$ & $7.91 \pm 0.48^{\dagger}$ & $8.86 \pm 0.57^{\dagger}$ \\
\hline Hospitalization & $0.15 \pm 0.02$ & $0.16 \pm 0.03$ & $0.17 \pm 0.04$ & $0.21 \pm 0.05$ \\
\hline ER visits & $0.28 \pm 0.03$ & $0.38 \pm 0.04^{*}$ & $0.42 \pm 0.06^{* *}$ & $0.51 \pm 0.07^{* * *}$ \\
\hline General practitioner/family practitioner visits & $2.25 \pm 0.10$ & $2.71 \pm 0.14^{* * *}$ & $3.06 \pm 0.19^{\dagger}$ & $3.50 \pm 0.23^{\dagger}$ \\
\hline Neurologist visits & $0.09 \pm 0.01$ & $0.16 \pm 0.02^{* *}$ & $0.21 \pm 0.04^{\dagger}$ & $0.34 \pm 0.06^{\dagger}$ \\
\hline Psychiatrist visits & $0.25 \pm 0.06$ & $0.29 \pm 0.08$ & $0.29 \pm 0.10$ & $0.40 \pm 0.14$ \\
\hline Psychologist/therapist visits & $0.33 \pm 0.06$ & $0.69 \pm 0.16^{* *}$ & $0.60 \pm 0.18^{*}$ & $0.83 \pm 0.26^{* *}$ \\
\hline Number of people with at least one HCP visit & $0.94 \pm 0.01$ & $0.97 \pm 0.01^{* * *}$ & $0.97 \pm 0.01^{* *}$ & $0.96 \pm 0.01$ \\
\hline $\begin{array}{l}\text { Number of people with at least one } \\
\text { hospitalization }\end{array}$ & $0.09 \pm 0.01$ & $0.10 \pm 0.01$ & $0.13 \pm 0.02$ & $0.12 \pm 0.02$ \\
\hline Number of people with at least one ER visit & $0.17 \pm 0.01$ & $0.22 \pm 0.02^{* *}$ & $0.22 \pm 0.02^{*}$ & $0.27 \pm 0.03^{\dagger}$ \\
\hline $\begin{array}{l}\text { Number of people with at least one } \\
\text { general/family practitioner visit }\end{array}$ & $0.78 \pm 0.02$ & $0.81 \pm 0.02^{*}$ & $0.84 \pm 0.02^{* *}$ & $0.84 \pm 0.02^{*}$ \\
\hline $\begin{array}{l}\text { Number of people with at least one neurologist } \\
\text { visit }\end{array}$ & $0.06 \pm 0.01$ & $0.11 \pm 0.01^{* * *}$ & $0.13 \pm 0.02^{\dagger}$ & $0.22 \pm 0.03^{\dagger}$ \\
\hline $\begin{array}{l}\text { Number of people with at least one psychiatrist } \\
\text { visit }\end{array}$ & $0.06 \pm 0.01$ & $0.07 \pm 0.01$ & $0.08 \pm 0.02$ & $0.09 \pm 0.02^{*}$ \\
\hline $\begin{array}{l}\text { Number of people with at least one } \\
\text { psychologist/therapist visit }\end{array}$ & $0.06 \pm 0.01$ & $0.08 \pm 0.01^{*}$ & $0.09 \pm 0.02^{*}$ & $0.12 \pm 0.02^{\dagger}$ \\
\hline
\end{tabular}

Generalized linear models were used for multivariable analyses

$E R$ Emergency room, $H C P$ healthcare provider, $H R U$ healthcare resource utilization, $S E$ standard error $p$ values for 1-3 MHDs vs. other migraine subgroups: ${ }^{*} p<0.05,{ }^{* *} p<0.01,{ }^{* * *} p<0.001,{ }^{\dagger} p<<0.0011$

study adds to the literature by including a large subgroup of migraineurs who reported experiencing low-frequency migraine (1-3 MHDs) as the reference category against which the migraineurs experiencing more frequent MHDs were compared.

The findings from the current study suggest that when compared with low-frequency episodic migraine (1-3 MHDs), intermediate-frequency episodic, high-frequency episodic, and chronic migraine ( $\geq 4$ MHDs) impose a greater burden on the individual and the healthcare system. This highlights the need for more effective steps to reduce the burden of migraine in individuals experiencing $\geq 4$ MHDs. "Lifting the Burden: the global campaign to reduce the burden of headache" by the World Health Organization also recognizes that headache disorders inflict pain and disability and that implementing relevant healthcare solutions would help relieve these burdens [28]. The solution to address migraine-related burden in most countries is education, as lack of awareness and proper medical training often hampers delivery of appropriate care [29]. 


\section{Limitations}

The NHWS is a panel-based survey, and while the panel administrators attempted to have samples that mirror the population as closely as possible (i.e., age and gender quotas for NHWS sampling), there could be a bias in the estimates, whereby there are differences between the population in each country and the panel population. This would most likely be among segments of the population who do not have a ready access to the Internet. The self-reported nature of the NHWS is also associated with potential corresponding biases, such as inaccurate recall and false reporting (whether intentional or unintentional). However, the consumer panel used validated self-report scales that were designed to minimize or eliminate intentional false reporting. Considering the larger sample size and the mode of administration of the questionnaire (either e-mail or phone), the study has used SF-6D or SF-12v2 questions for ease of administration and to reduce respondent fatigue without compromising on data reliability.[30, 31]. However, the short form versions cover all aspects and are derived from the SF-36 questionnaire. The difficulty in answering the survey questions over the telephone or e-mail in hospitalized or very sick individuals as well as the difficulty of a respondent in recalling the burden are limitations even in the context of administering short form versions for the survey. The study does not capture mental illnesses because the respondents might have suffered from mental illnesses due to migraine, which could have subsequently impacted the patients' HRQoL and HRU [32, 33]. Indeed, the increased psychologist and psychiatrist visits could be partially attributed to mental illnesses.

\section{CONCLUSION}

Although respondents experiencing 1-3 MHDs were found to be more prevalent than those experiencing $\geq 4$ MHDs (i.e., 4-7, 8-14, and $\geq 15$ MHDs), the incremental burden due to migraine in terms of reduced HRQoL, greater work productivity loss and activity impairment, and increased HRU was higher among those in the $\geq 4$ MHDs subgroups than among those in the $\leq 3$ MHDs subgroup in the EU5. The findings from this study suggest that respondents experiencing $\geq 4$ MHDs, chronic migraineurs ( $\geq 15$ MHDs) in particular, should be treated more effectively to reduce migraine frequency and lessen the burden of migraine in the EU5. Preventing the conversion of episodic into chronic migraine should also be an important consideration for protecting migraineurs from increased burden.

\section{ACKNOWLEDGEMENTS}

We thank the participants of the study and the peer reviewers of this paper for their feedback.

Funding. This study, including the Rapid Service Fee, was sponsored by Novartis Pharma AG, Switzerland.

Authorship. All named authors meet the International Committee of Medical Journal Editors (ICMJE) criteria for authorship for this article, take responsibility for the integrity of the work as a whole, and have given their approval for this version to be published.

Authorship Contribution. MJD, SG, JF, AKL, and PV conceived and designed the study. MJD and SG analyzed the data. MJD, SG, JF, AKL, and PV interpreted the results and helped write the paper. All authors read and approved the final manuscript.

Medical Writing, Editorial, and other Assistance. The authors acknowledge Ramu Periyasamy, PhD, Indegene Pvt Ltd., for his assistance with literature review and writing the manuscript.

Disclosures. Shaloo Gupta is an employee of Kantar. Michael J. Doane was an employee of Kantar Health when the study was conducted but his current affiliation is Alkermes, Inc. Pamela Vo and Juanzhi Fang are employees of Novartis Pharma AG that funded this study. Annik K. Laflamme worked at Novartis Pharms 
AG at the time of the study and is currently affiliated with Sobi ${ }^{\mathrm{TM}}$ Swedish Orphan Biovitrum AG.

Compliance with Ethics Guidelines. The NHWS received approval from the Pearl Institutional Review Board. All the NHWS respondents provided informed consent electronically prior to their participation.

Data Availability. Survey data used in this study can be made available for non-commercial research and validation purposes only, upon request.

Open Access. This article is licensed under a Creative Commons Attribution-NonCommercial 4.0 International License, which permits any non-commercial use, sharing, adaptation, distribution and reproduction in any medium or format, as long as you give appropriate credit to the original author(s) and the source, provide a link to the Creative Commons licence, and indicate if changes were made. The images or other third party material in this article are included in the article's Creative Commons licence, unless indicated otherwise in a credit line to the material. If material is not included in the article's Creative Commons licence and your intended use is not permitted by statutory regulation or exceeds the permitted use, you will need to obtain permission directly from the copyright holder. To view a copy of this licence, visit http://creativecommons.org/licenses/bync/4.0/.

\section{REFERENCES}

1. Olesen J, Headache Classification Subcommittee of the International Headache Society. The international classification of headache disorders: 2nd edition. Cephalalgia. 2004;24(Suppl.):9-10.

2. Buse DC, Rupnow MFT, Lipton RB. Assessing and managing all aspects of migraine: migraine attacks, migraine-related functional impairment, common comorbidities, and quality of life. Mayo Clin Proc. 2009;84:422-35.
3. Cady RK. The future of migraine: beyond just another pill. Mayo Clin Proc. 2009;84:397-9.

4. Woldeamanuel YW, Cowan RP. Migraine affects 1 in 10 people worldwide featuring recent rise: a systematic review and meta-analysis of communitybased studies involving 6 million participants. J Neurol Sci. 2017;372:307-15.

5. World Health Organization. Headache disorders. $2016 . \quad$ https://www.who.int/mediacentre/ factsheets/fs277/en/. Accessed 11 Sep 2018

6. Vos T, Barber RM, Bell B, et al. Global, regional, and national incidence, prevalence, and years lived with disability for 301 acute and chronic diseases and injuries in 188 countries, 1990-2013: a systematic analysis for the Global Burden of Disease Study 2013. Lancet. 2015;386:743-800.

7. Brown JS, Neumann PJ, Papadopoulos G, et al. Migraine frequency and health utilities: findings from a multisite survey. Value Health. 2008;11: 315-21.

8. Vo P, Fang J, Bilitou A, et al. Patients' perspective on the burden of migraine in Europe: a cross-sectional analysis of survey data in France, Germany, Italy, Spain, and the United Kingdom. J Headache Pain. 2018;19:82.

9. Lipton RB, Bigal ME, Diamond M, et al. Migraine prevalence, disease burden, and the need for preventive therapy. Neurology. 2007;68:343-9.

10. Blumenfeld A, Varon S, Wilcox T, et al. Disability, HRQOL and resource use among chronic and episodic migraineurs: results from the International Burden of Migraine Study (IBMS). Cephalalgia. 2011;31:301-15.

11. Wang S-J, Wang P-J, Fuh J-L, et al. Comparisons of disability, quality of life, and resource use between chronic and episodic migraineurs: a clinic-based study in Taiwan. Cephalalgia. 2013;33:171-81.

12. Berra E, Sances G, De Icco R, et al. Cost of chronic and episodic migraine: a pilot study from a tertiary headache centre in northern Italy. J Headache Pain. $2015 ; 16: 50$.

13. Raggi A, Giovannetti AM, Schiavolin S, et al. Validating the Migraine-Specific Quality of Life Questionnaire v2.1 (MSQ) in Italian inpatients with chronic migraine with a history of medication overuse. Qual Life Res. 2014;23:1273-7.

14. Radtke A, Neuhauser H. Prevalence and burden of headache and migraine in Germany. Headache. 2009;49:79-89. 
15. Charlson ME, Pompei P, Ales KL, et al. A new method of classifying prognostic comorbidity in longitudinal studies: development and validation. J Chronic Dis. 1987;40:373-83.

16. Maruish ME, editor. User's manual for the SF-36v2 Health Survey. 3rd ed. Lincoln, RI: QualityMetric Incorporated; 2011.

17. Montazeri A, Vahdaninia M, Mousavi SJ, et al. The 12-item medical outcomes study short form health survey version 2.0 (SF-12v2): a population-based validation study from Tehran, Iran. Health Qual Life Outcomes. 2011;9:12. https://doi.org/10.1186/ 1477-7525-9-12.

18. Brazier J, Roberts J, Deverill M. The estimation of a preference-based measure of health from the SF-36. J Health Econ. 2002;21:271-92.

19. Brazier J, Rowen D, Hammer J. Revised SF-6D scoring programmes: a summary of improvements. Patient Rep Outcomes Newsl. 2008;40:14-5.

20. Herdman M, Gudex C, Lloyd A, et al. Development and preliminary testing of the new five-level version of EQ-5D (EQ-5D-5L). Qual Life Res. 2011;20: 1727-36.

21. Reilly MC, Zbrozek AS, Dukes EM. The validity and reproducibility of a work productivity and activity impairment instrument. PharmacoEconomics. 1993;4:353-65.

22. Reilly Associated. WPAI scoring. https://www. reillyassociates.net/WPAI_Scoring.html. Accessed 25 Jul 2019.

23. Gupta S, Goren A, Phillips AL, et al. Self-reported burden among caregivers of patients with multiple sclerosis. Int J MS Care. 2012;14:179-87.

24. daCosta DiBonaventura M, Gupta S, McDonald M, et al. Evaluating the health and economic impact of osteoarthritis pain in the workforce: results from the National Health and Wellness Survey. BMC Musculoskelet Disord. 2011;12:83.
25. Goren A, Liu X, Gupta S, et al. Quality of life, activity impairment, and healthcare resource utilization associated with atrial fibrillation in the US National Health and Wellness Survey. PLoS ONE. 2013;8:e71264.

26. Ayzenberg I, Katsarava Z, Sborowski A, et al. Headache-attributed burden and its impact on productivity and quality of life in Russia: structured healthcare for headache is urgently needed. Eur J Neurol. 2014;21:758-65.

27. Edmeads J, Mackell JA. The economic impact of migraine: an analysis of direct and indirect costs. Headache J Head Face Pain. 2002;42:501-9.

28. World Health Organization (WHO). Lifting the burden: the global campaign to reduce the burden of headache. https://www.who.int/mental_health/ neurology/headache/en/. Accessed 21 Mar 2019

29. Steiner TJ. Lifting the burden: the global campaign to reduce the burden of headache worldwide. J Headache Pain. 2005;6:373-7.

30. Kost RG, Correa da Rosa J. Impact of survey length and compensation on validity, reliability, and sample characteristics for Ultrashort-, Short-, and Long-Research Participant Perception Surveys. J Clin Transl Sci. 2018;2:31-7.

31. The iConnect Consortium, Sahlqvist S, Song Y, et al. Effect of questionnaire length, personalisation and reminder type on response rate to a complex postal survey: randomised controlled trial. BMC Med Res Methodol. 2011;11:62.

32. Jette N, Patten S, Williams J, et al. Comorbidity of migraine and psychiatric disorders-a National Population-Based Study. Headache J Head Face Pain. 2008;48:501-16.

33. Merikangas KR. Migraine and psychopathology: results of the Zurich Cohort Study of young adults. Arch Gen Psychiatry. 1990;47:849. 